\title{
Automatic segmentation, detection and quantification of coronary artery stenoses on CTA
}

\author{
Rahil Shahzad • Hortense Kirişli • Coert Metz • \\ Hui Tang • Michiel Schaap • Lucas van Vliet • \\ Wiro Niessen $\cdot$ Theo van Walsum
}

Received: 12 April 2013/Accepted: 28 July 2013/Published online: 8 August 2013

(C) Springer Science+Business Media Dordrecht 2013

\begin{abstract}
Accurate detection and quantification of coronary artery stenoses is an essential requirement for treatment planning of patients with suspected coronary artery disease. We present a method to automatically detect and quantify coronary artery stenoses in computed tomography coronary angiography. First, centerlines are extracted using a two-point minimum cost path approach and a subsequent refinement step. The resulting centerlines are used as an initialization for lumen segmentation, performed using graph cuts. Then, the expected diameter of the healthy lumen is estimated by applying robust kernel regression to the coronary artery lumen diameter profile. Finally, stenoses are detected and quantified by computing the difference between estimated and expected diameter profiles. We evaluated our method using the data provided in the Coronary Artery Stenoses Detection and Quantification Evaluation Framework. Using 30 testing datasets, the method achieved a detection sensitivity of $29 \%$ and a positive predictive value (PPV) of $24 \%$ as compared to
\end{abstract}

Rahil Shahzad and Hortense Kirişli contributed equally to this research.

R. Shahzad $(\varangle) \cdot$ H. Tang $\cdot$ L. van Vliet $\cdot$ W. Niessen Quantitative Imaging Group, Department of Imaging Science and Technology, Faculty of Applied Science, Delft University of Technology, Delft, The Netherlands

e-mail: R.Shahzad@tudelft.nl

R. Shahzad · H. Kirişli · C. Metz · H. Tang · M. Schaap ·

W. Niessen · T. van Walsum

Biomedical Imaging Group Rotterdam, Department of

Radiology and Medical Informatics, Erasmus MC, Rotterdam,

The Netherlands

Present Address:

M. Schaap

HeartFlow, Inc., Redwood City, CA, USA quantitative coronary angiography (QCA), and a sensitivity of $21 \%$ and a PPV of $23 \%$ as compared manual assessment based on consensus reading of CTA by 3 observers. The stenoses degree was estimated with an absolute average difference of $31 \%$, a root mean square difference of $39.3 \%$ when compared to QCA, and a weighted kappa value of 0.29 when compared to CTA. A Dice of 68 and 65 $\%$ was reported for lumen segmentation of healthy and diseased vessel segments respectively. According to the ranking of the evaluation framework, our method finished fourth for stenosis detection, second for stenosis quantification and second for lumen segmentation.

Keywords Centerline extraction - Lumen segmentation $\cdot$ Calcium suppression $\cdot$ QCA

\section{Introduction}

Coronary artery disease (CAD) is one of the leading causes of death worldwide [1,2]. CAD induces plaque build-up in the coronary arteries, which may cause luminal narrowing also known as stenosis. Stenoses may induce myocardial infarction; it is therefore crucial to detect CAD at an early stage.

Many diagnostic tests are available for detection of CAD [3]. At present, invasive coronary angiography (ICA) is the reference standard imaging technique for diagnosing $\mathrm{CAD}$ and quantitative coronary angiography (QCA) is used to quantify the degree of stenosis. However, ICA is an invasive procedure and is limited by its projective nature. Computed tomography coronary angiography (CTA) on the other hand, is increasingly used to assess CAD and has the advantage over ICA of being non-invasive. Furthermore, it provides high resolution three-dimensional (3D) 
images of the coronary arteries. In addition to the detection and quantification of coronary artery stenoses, CTA can also provide additional information regarding the type of plaque (calcified, mixed or soft) [4]. It has been shown that CTA scans can be used to accurately identify the presence and severity of the stenoses in comparison to ICA in [5]. However, interpreting CTA images for the purpose of stenosis detection and quantification requires considerable experience to prevent underestimating or overestimating obstructive plaque lesions [6], and is therefore a tedious task. Whereas we are focusing on stenosis grading, similar approaches may be relevant for CT FFR as well, where the combination of computational flow models and accurate segmentations may predict the hemodynamic significance of a lesion [7, 8].

Consequently, the number of publications presenting and/or evaluating (semi-) automatic coronary artery stenosis detection and quantification methods on cardiac CTA have increased in recent years. An algorithm evaluation framework dedicated to this problem has been introduced in 2012 (http://coronary.bigr.nl/stenoses) [9].

Different approaches have been proposed to address the challenge of (semi-) automatically detecting and quantifying stenoses. These methods can be categorised into two groups: (1) methods that depend on accurate lumen segmentation to compute/estimate healthy and diseased lumen diameters in order to quantify stenoses [10-15] and (2) methods that use image features or pattern recognition approaches to detect stenoses [16-18].

In this study, we present an automatic method for coronary artery lumen segmentation, stenosis detection and quantification. The method aims at facilitating and supporting the interpretation of cardiovascular CTA data by radiologists. The method has been evaluated through the Coronary Artery Stenoses Detection and Quantification Evaluation Framework [9].

The remainder of this paper is organized as follows. In "Materials and methods" section, we describe the data used, our method and the parameter value selection. "Results" section is dedicated to the evaluation of our approach. Results of our approach are discussed in "Discussion" section, as well as the limitations and possibilities for future studies. Conclusions are given in "Conclusion" section.

\section{Materials and methods}

Imaging data

The data used for this study was obtained from the publicly available Coronary Artery Stenoses Detection and Quan- tification Evaluation Framework (http://coronary.bigr.nl/ stenoses). The datasets provided by this framework were retrospectively acquired in three university medical centers (Erasmus MC, University Medical Center, Rotterdam, the Netherlands; University Medical Center Utrecht, Utrecht, the Netherlands; and Leiden University Medical Center, Leiden, the Netherlands). The patients underwent both CTA and QCA examinations. Below, we provide information about the image acquisition, data selection and reference standards. Additional information can be found on the website (http://coronary.bigr.nl/stenoses/about.php).

The CTA data was acquired on : (1) a dual-source CT scanner (Somatom Definition, Siemens, Forchheim, Germany) at the Erasmus MC, (2) a 64-slice CT scanner (Brillance 64, Philips Medical Systems, Best, the Netherlands) at the UMCU, and (3) a 320-slice CT scanner (Aquilion ONE 320, Toshiba Medical Systems, Tokyo, Japan) at the LUMC. A non-enhanced CT scan was performed before the CTA; the total calcium score for each patient was calculated using a dedicated software in each center.

A single image volume per patient was used, reconstructed at the mid-to-end diastolic phase $(350 \mathrm{~ms}$ before the next R-wave or at $65-70 \%$ of the R-R interval), with either retrospective (Siemens and Philips data) or prospective (Toshiba data) ECG gating.

Sixteen patients, distributed over five calcium categories in order to have a representative population that undergoes CTA examination, were selected in each of the three centers, resulting in 48 datasets. The calcium categories [19] are defined as follows: no calcium (11 patients, $23 \%$ ), between 0.1 and 10 ( 6 patients, $10 \%)$, between 11 and 100 (14 patients, $31 \%$ ), between 101 and 400 (11 patients, $23 \%$ ), and above 400 Agatston score (6 patients, $12 \%$ ). The population has a mean age of $58.76 \pm 8.71(41-80)$ years old and consists of 30 males $(63 \%)$.

Eighteen of the 48 CTA images, together with the CTA and QCA reference standards, were made available for training; the remaining 30 datasets were used for testing the algorithms. For testing, only the CTA images were made available. The distribution of patients over the two sets is explained in detail in recent work of Kirisli et al. [9].

Three independent experienced observers from Erasmus MC, University Medical Center Rotterdam, analysed the CTA datasets and provided the ground truth via consensus reading. A dedicated tool implemented in MeVisLab (http://www.mevislab.de) was used by the observers for the annotations. QCA analysis was performed by an independent observer blinded to the CTA results. The ground truth data consists of quantification and stenosis detection on both the CTA and ICA datasets, as well as lumen segmentation on the CTA dataset (Fig. 1). 
Fig. 1 Extracted initial centerlines for one of the datasets. a Right branch, b left branch
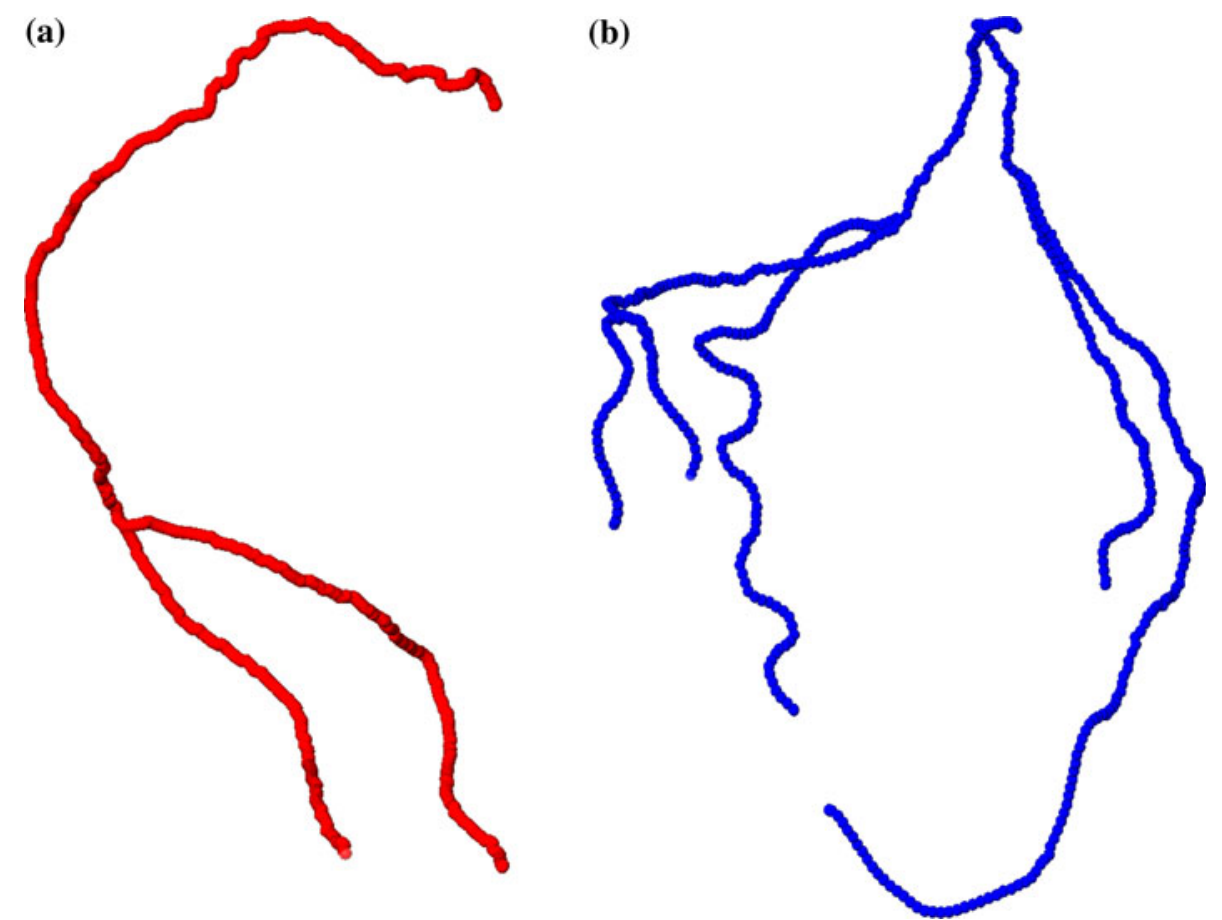

Method

The method described in this paper consists of the following steps: (1) centerlines are extracted using predefined start and end points in the arteries ("Centerline extraction" section), (2) bifurcation points of the extracted vascular tree are detected and the centerlines are subsequently divided into segments ("From centerlines to segments" section) (3) coronary artery lumen segmentation is performed using the centerline segments as initialization ("Lumen segmentation" section), and (4) stenoses are detected and quantified using an area based approach ("Stenosis detection and quantification" section).

\section{Centerline extraction}

The centerlines of the coronary artery tree are extracted as follows.

First, for each branch of the coronary artery tree, an initial centerline is obtained, by applying the minimum cost path extraction method presented in [20]. A 3D path with minimum cost is found between two manually placed seed points in the coronary arteries, located at the ostium and at the distal end of each coronary artery. The cost image $C_{v}(x)$, (with $x$ a location in the image) used for centerline extraction is based on a multi-scale vesselness measure $V(x)$ [21] and a sigmoid like intensity threshold function $T(x)$ [20], and is defined as:

$C_{v}(x)=\frac{1}{V(x) T(x)+\epsilon}$, where $\epsilon$ is a small positive value introduced to avoid singularities (see "Parameter selection" section, for parameter values).

However, the initial centerlines obtained are inaccurate at the bifurcations and at calcified locations, where the centerline is attracted towards the calcified part of the vessel due to relatively low cost values inside the calcifications. Therefore, the centerlines are refined in a subsequent step.

In this second step, calcium lesions within the artery are suppressed, based on the intensity profile of the contrast material along the initially extracted centerline. In the ideal case, i.e. a healthy vessel presenting no calcified plaque, the intensity profile is a smooth curve with a gradual decrease in intensity along the artery (see Fig. 2a) [22, 23]. But, in the case that a centerline passes through a calcified plaque, the intensity profile presents a spike, indicating the presence of a high intensity object along the centerline path. In the case where the contrast material is not evenly distributed throughout the artery, intensity variations not corresponding to calcified plaques may also appear in the intensity profile. In order (1) to differentiate true calcium objects from noise, and (2) to estimate the intensity value of the contrast material within the coronary artery, we apply a cubic fit to the intensity profile of the initially extracted centerline. Figure 2 shows examples of intensity profiles along different artery segments.

Given an intensity profile for centerline points $x \in X$, where $X$ is the set of centerline points, point $x$ is assigned as belonging to a calcium object if it obeys the condition: 

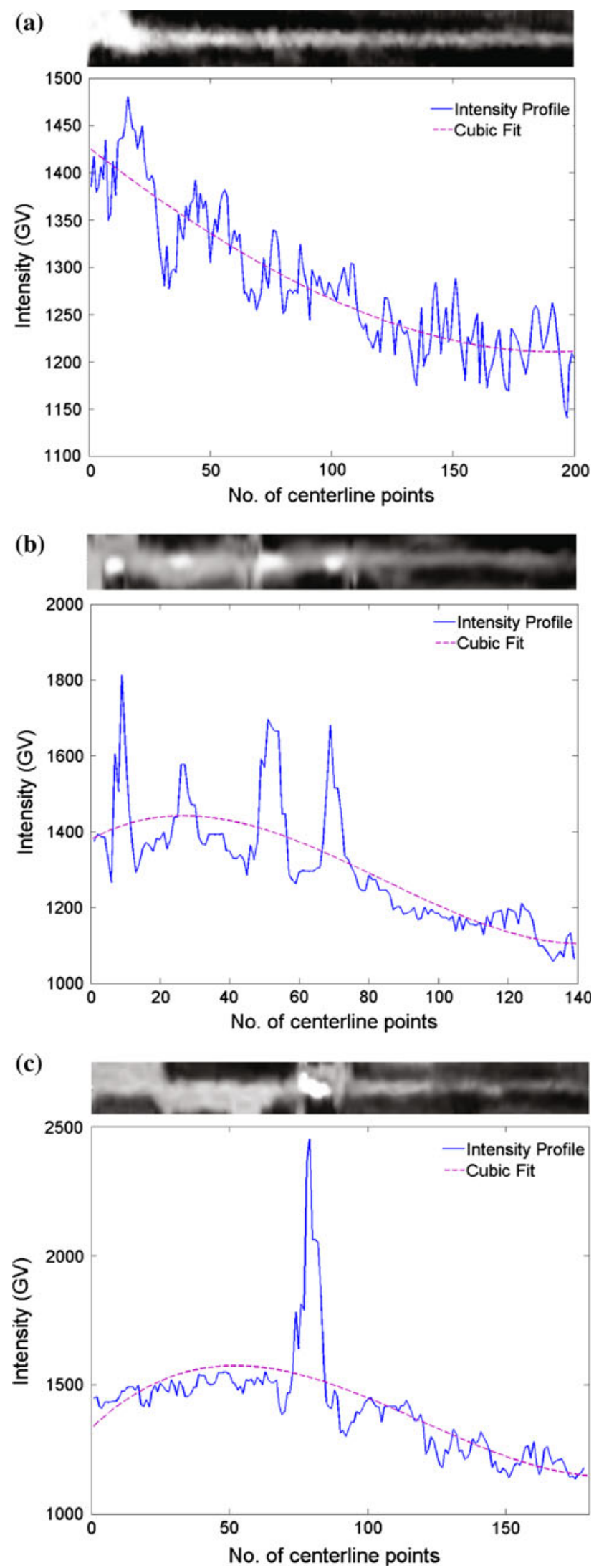

Fig. 2 Plot of the intensity (in GV) as a function of position along the centerline and the corresponding CMPR images for three coronary artery segments. a Segment without calcium objects, b segment with multiple calcium objects, c segment with a high density calcium object

$I(x)-\left.F(x)\right|_{x \in X} \geq T_{c a}$,

where $I(x)$ is the CTA image intensity at position $x$ along the centerline, $F(x)$ is the value at position $x$ based on a fitted cubic polynomial and $T_{c a}$ is a predefined threshold value (see "Parameter selection" section).

All the centerline points $x$ which satisfy Eq. 2 are treated as seedpoints to initialize a region growing segmentation with a 3D 6-neighbourhood relation. If a connected voxel has an intensity greater than equal to $\max _{x \in X}(F(x))$, it is classified as belonging to a calcium object. The segmented calcium object is suppressed by setting its intensity value to 1,024 grayscale value $(\mathrm{GV})(1,024 \mathrm{GV}=0$ Hounsfield unit). Figure 3 shows an example where a calcium lesion is suppressed.

Subsequently, to move the centerlines running close to the border of the lumen towards the vessel center, we generate a stack of multi-planar reformatted (MPR) images, i.e. a stack of images perpendicular to the initial centerline. We then apply a minimum cost path approach to this image stack, as proposed by Tang et al. [24]. Using a modified cost image $C_{m v}(x)$ based on both $V(x)$ and a medialness measure $M(x)$ [25], defined as:

$C_{m v}(x)=\frac{1}{V(x) M(x)+\epsilon}$.

Figure 4 shows an example of an MPR image generated using the refined centerlines.

\section{From centerlines to segments}

To extract the lumen centerlines ("Centerline extraction" section), a unique starting point (located in the ostium) and multiple end points are used. As a consequence, the extracted centerlines present multiple overlapping paths. At locations where a vessel bifurcates, a sudden drop in the vessel diameter occurs. This influences the stenoses detection and quantification step ("Stenosis detection and quantification" section) which is based on cross-sectional vessel area. In order to facilitate further processing, we first filter the centerline points using Mean Shift filtering [26], such that matching co-linear centerlines are closer to each other. Subsequently, we can merge centerlines representing the same segment, and detect bifurcations at locations where centerlines are diverging. 
Fig. 3 A random crosssectional image slice through a calcium lesion. a Before calcium suppression, b after calcium suppression
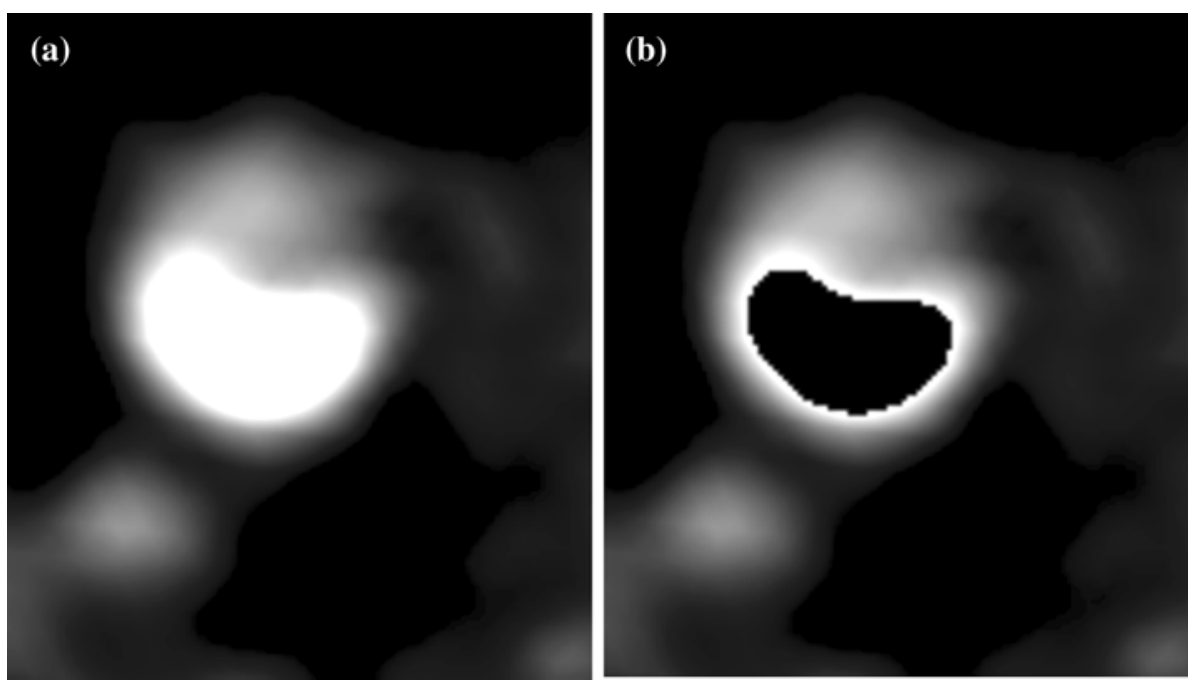
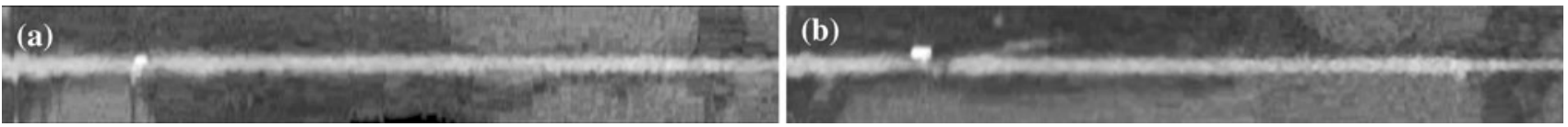

Fig. 4 a CMPR image before calcium object suppression and centerline refinement, b CMPR image after calcium object suppression and centerline refinement. It can be observed from $\mathbf{b}$ that the calcium object is much better separated from the lumen

For the filtering, each centerline $S_{i=1 \ldots m}$ (with $m$ the total number of centerline segments) is equidistantly resampled to a set of spatial points $\left\{\mathbf{x}_{1} \ldots \mathbf{x}_{n_{i}}\right\}$ with $n_{i}$ the number of points of the centerline $S_{i}$. We then filter the centerlines using the approach proposed by van Walsum et al. [27], and subsequently build a graph from these filtered centerlines representing the coronary tree structure.

The Mean Shift filtering algorithm used is represented as follows:

$\mathbf{x}_{k l}^{\tau+1}=\sum_{i j} \frac{c_{k l, i j} \phi_{k l, i j} G_{s}\left(\left|\mathbf{x}_{k l}^{\tau}-\mathbf{x}_{i j}\right|\right)}{\sum_{i^{\prime} j^{\prime}} c_{k l, i^{\prime} j^{\prime}} \phi_{k l, i^{\prime} j^{\prime}} G_{s}\left(\left|\mathbf{x}_{k l}^{\tau}-\mathbf{x}_{i^{\prime} j^{\prime}}\right|\right)} \mathbf{x}_{i j}$

which states that in each iteration, point $x$ is replaced with a weighted average of the points of all centerlines. The subscripts $i j, k l$ represent the $j$ th point on the $i$ th centerline and the $l$ th point on the $k$ th centerline. Convergence is reached if the distance between $\mathbf{x}^{\tau}$ and $\mathbf{x}^{\tau+1}$ is less then a small threshold $(\delta)$.

The weights in Eq. 4 have three components. $c_{k l, i j}$ is a correspondence term, based on connectivities between points of different centerlines. It uses Dijkstra graph search algorithm [28] to determine the set of connections $D_{k i}=\left\{d_{k l, i j}\right\}$ between centerlines $S_{k}$ and $S_{i}$, with $d_{k l, i j}$ a connection between $\mathbf{x}_{k l}$ and $\mathbf{x}_{i j}$. $c_{k l, i j}$ is defined as:

$c_{k l, i j}=\left\{\begin{array}{cl}0 & \text { if } d_{k l, i j} \notin D_{k i} \\ \left|\left\{d_{k l, i j^{\prime}}\right\}\right|^{-1} & \text { if } d_{k l, i j} \in D_{k i}\end{array}\right.$

with $|\cdot|$ the cardinality of the set. $\phi_{k l, i j}$ decreases the weights for points with differently oriented tangent, and prevents averaging over bifurcations. It is defined as:

$\phi_{k l, i j}=G_{\phi}\left(\operatorname{acos}\left(\mathbf{t}_{k l} \cdot \mathbf{t}_{i j}\right)\right)$,

with $G_{\phi}$ the Gaussian kernel with standard deviation $\sigma_{\phi}$ and $\mathbf{t}_{i j}$ the (normalized) tangents vector at $\mathbf{x}_{i j}$. This orientation factor is 1 when the tangents are parallel, and less then 1 when the tangents diverge.

$G_{s}$ is the Gaussian weighted distance with a standard deviation of $\sigma_{s}$ to decrease the influence of points far away.

Application of Eq. 4 to all points $\mathbf{x}_{i j}$ of all centerlines $S_{i}$ yields shifted centerlines $S_{i}^{\prime}$. The shifting process is followed by combining these shifted centerlines into a directed graph representation. To this end, all centerlines are added to a graph consecutively, where the initial graph is empty. For each centerline to be added, the overlapping parts with the existing graph are determined, and the overlapping parts are merged in the graph's data structure. For each of the non-overlapping parts, new edges are created in the graph. After merging all the centerlines into the graph, each path and node (bifurcation point) of the graph structure contains references to the corresponding parts of the (shifted) centerlines. Figure 5 shows an example of a segmented and labelled coronary artery tree, where different colors indicate different segments and the white balls represent the bifurcation points, start points and the end points. 
Fig. 5 Result of automatic bifurcation detection in which different colors represent different segments. a Right coronary artery tree, $\mathbf{b}$ left coronary artery tree
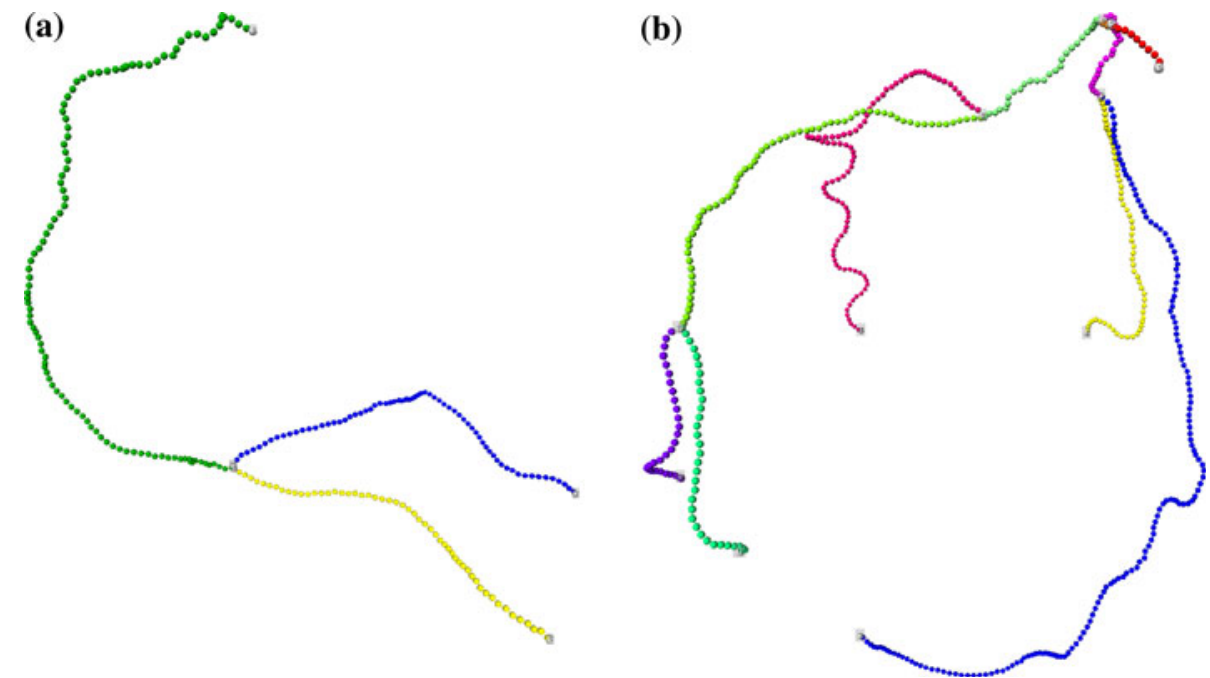

\section{Lumen segmentation}

The coronary lumen is segmented using a method combining graph-cuts and robust kernel regression [29]. The method is applied segment wise and uses the refined centerline as initialization. The segmentation process is performed on the MPR image stack.

The graph-cuts method uses an application specific unary term based on the image intensities of the centerlines (voxel likelihood) and a binary term based on the image gradient magnitude (edge term). Essentially, the voxel likelihood term assigns high foreground weights and low background weights to voxels with similar intensities as the centerline intensities, and vice versa for voxels that have dissimilar intensity values. The voxel likelihood term is defined as:

$$
\begin{aligned}
\operatorname{Pr}\left(I_{x} \mid f_{x}=1\right)= & -0.5\left(0.75-0.25 \operatorname{erf}\left(\frac{D_{x}-T_{\text {in }}}{\sigma_{i}}\right)\right) \\
& \times\left(\operatorname{erf}\left(\frac{D_{x}-T_{\text {out }}}{\sigma_{i}}\right)-1\right)
\end{aligned}
$$

with $D_{x}=\left|I_{x}-\hat{I}_{x^{\prime}}\right|$ the absolute difference between the intensity of the voxel $\left(I_{x}\right)$ and the local intensity estimate $\left(\hat{I}_{x^{\prime}}\right)$, $T_{i n}$ the threshold parameter for intensities within the lumen, $T_{\text {out }}=\lambda\left(\operatorname{Mean}(\mathrm{I})-I_{o}\right)$ the difference between the mean intensity I along the centerline and the intensity outside $I_{o}$, i.e the threshold parameter for intensities outside the lumen. Figure $6 \mathrm{~b}$ shows an axial slice after the application of Eq. 7.

The edge term uses the gradient magnitude at the boundary of two voxels. A higher value corresponds to a high probability of the voxel label being switched, between lumen and background. The weight of a label switch between voxel $x$ and $y$ is defined as:

$w_{x, y}=-\log \left(1-\exp \left(\frac{-|\nabla I|^{2}(x, y)}{2 \sigma_{g}^{2}}\right)\right)$.
After the graph-cut segmentation each voxel is assigned to the lumen or non-lumen (see Fig. 6c). Because this segmentation is discrete and because it can contain outliers, the segmentation is smoothed and outliers are removed with a robust kernel regression approach. The graph-cut lumen boundary is first described in a cylindrical coordinate system by finding, in each cross-section, the intersection between rays, sampled at fixed angles from the centerline. This representation is subsequently smoothed with a robust kernel regression approach, ensuring that both outliers are removed and a smooth boundary representation is obtained (see Fig. 6d).

\section{Stenosis detection and quantification}

From the coronary artery lumen segmentation (per segment of the coronary artery tree), the cross-sectional area $A_{i}$ of the vessel is computed at every position $i$ along the vessel centerline, $i \in[1, n]$ with $n$ being the number of positions along the centerline. The radius is then derived as $r_{i}=\sqrt{A_{i} / \pi}$.

To compute the degree of stenosis, the radius of a healthy vessel is needed as a reference. We estimated the radius $\hat{r}$ of the healthy vessel by applying a robust weighted Gaussian kernel regression [30] to the 1D function $r$ describing the vessel radius along the centerline:

$\hat{r}_{i}=\frac{\sum_{i^{\prime}=1}^{n} N\left(i^{\prime} \mid i, \sigma_{i}\right) w_{i^{\prime}} r_{i^{\prime}}}{\sum_{i^{\prime}=1}^{n} N\left(i^{\prime} \mid i, \sigma_{i}\right) w_{i^{\prime}}}$,

where,

$\left[\begin{array}{c}w_{i}=N\left(r_{i} \mid r_{i}^{\max }, \sigma_{r}\right) \\ r_{i}^{\max }=\frac{\sum_{i^{\prime}=1}^{n} N\left(i^{\prime} \mid i, \sigma_{\max }\right) r_{i^{\prime}}}{\sum_{i^{\prime}=1}^{n} N\left(i^{\prime} \mid i, \sigma_{\max }\right)} \\ N\left(i^{\prime} \mid i, \sigma\right)=\frac{1}{\sigma \sqrt{2 \pi}} e^{-\frac{\left(i^{\prime}-i\right)^{2}}{2 \sigma^{2}}}\end{array}\right.$ 
Fig. 6 A cross-sectional image of a randomly selected coronary artery, presenting a calcium lesion and a side branch. a Input image, b resulting image after applying a lumen likelihood function, $\mathbf{c}$ binarized image after lumen segmentation using graph cuts (lumen bright, background black), $\mathbf{d}$ the resulting lumen segmentation (in white) after kernel regression
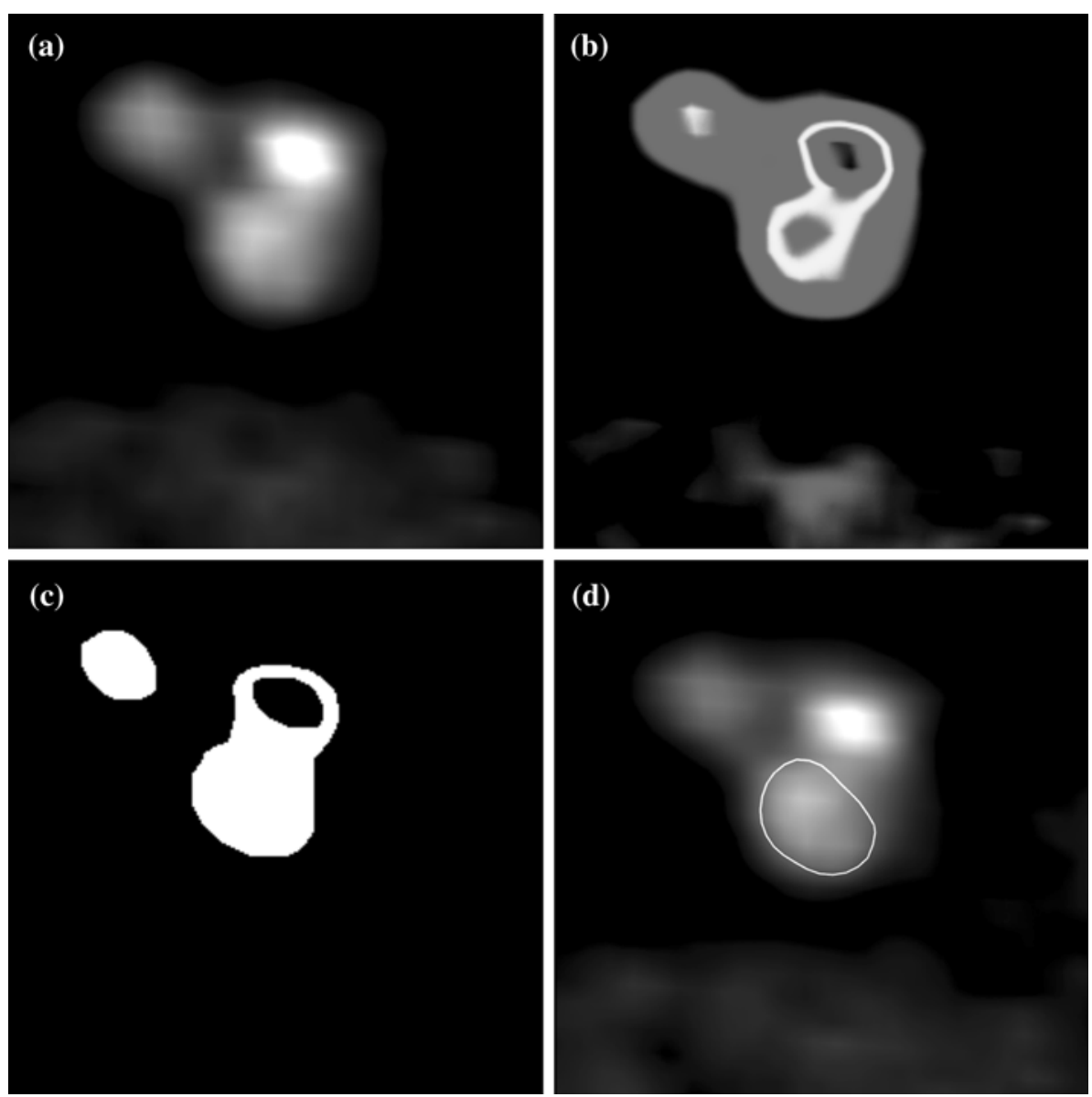

\section{Parameter selection}

Some of the parameters used in our method were optimized using the training datasets provided by the framework. For others, the values were chosen identical to our previous works The lower and upper scales for the multi-scale vesselness measure $\left(V_{x}\right)$ used in Eq. 1 and in Eq. 3 were set to 0.8 and $2 \mathrm{~mm}$, with 3 intermediate scales. The other parameters $\alpha, \beta, c$ (used in $V_{x}$ ), $w_{1}, w_{2}, w_{3}, a_{s}$ and $b_{s}$ (used in $T_{x}$ ) from Eq. 1 were taken from [20] and are presented in Table 1. The minimum and maximum scales for the medialness measure $\left(M_{x}\right)$ used in Eq. 3 were set to $0.5 \mathrm{~mm}$ and $2 \mathrm{~mm}$, the number of intermediate scale steps to 8 and the number of angles to 24. The value of $\epsilon$ in both equations was set to 0.0001 . The value of $T_{c a}$ in Eq. 2 was set to $200 \mathrm{GV}$. The CMPR images were generated at $0.5 \mathrm{~mm}$ slice spacing and with a cross-sectional area of

Table 1 Parameters used in computing the vesselness measure $V_{x}$ and the threshold function $T_{x}$

\begin{tabular}{llllllll}
\hline$\alpha$ & $\beta$ & $c$ & $w_{1}$ & $w_{2}$ & $w_{3}$ & $a_{s}$ & $b_{s}$ \\
\hline 0.5 & 0.4 & 230 & 0.99 & 0.10 & 0.10 & $1028 \mathrm{GV}$ & $965 \mathrm{GV}$ \\
\hline
\end{tabular}

$10 \times 10 \mathrm{~mm}^{2}$, and a voxel size of $0.1 \times 0.1 \times 0.5 \mathrm{~mm}^{3}$. The parameters used for lumen segmentation in "Lumen segmentation" section were taken from [29]. The value of $\sigma_{s}$ and $\delta$ in Eq. 4 were set to 0.5 and 0.01 respectively, $\sigma_{\phi}$ in Eq. 6 was set to 0.1. In Eqs. 9 and 10 of the stenoses detection/quantification, the parameter $\sigma_{x}$ (corresponding to centerline longitudinal distance) was set to $8, \sigma_{r}$ (corresponding to radius) to 0.25 , and $\sigma_{\max }$ to 200 .

As QCA is the reference standard, it was observed from the training experiments that the CTA derived measure slightly overestimates the degree of stenosis in the mild stenotic regions. This is probably due to the fact that QCA measurements are made in 2D and our method quantifies the stenoses in 3D on the CTA image. Therefore, we investigated the possibility to improve the quantification measure, correcting for this bias. We performed a few pilot experiments and found out that improved stenoses quantification matching between CTA and QCA can be achieved by applying an off-set value of -20 (represented as \%) to all lesions detected on CTA with a degree between 20 and $50 \%$ (Fig. 7).

Our method also overestimates the degree of stenosis on CTA in case of highly calcified lesions, due to the 
Fig. 7 Stenoses detection and quantification. a Shows the reconstructed lumen tree of a patient in which the red shade highlights narrowing of the lumen. b Various curves as a function of centerline position showing: the true radius (black), estimated radius (green), detected stenoses (blue) and the stenoses degree (black dotted). The cross on the stenosis in (a) corresponds to the vertical line at the $18 \mathrm{~mm}$ mark in (b). It can also be observed that two stenoses were detected and one of them was significant
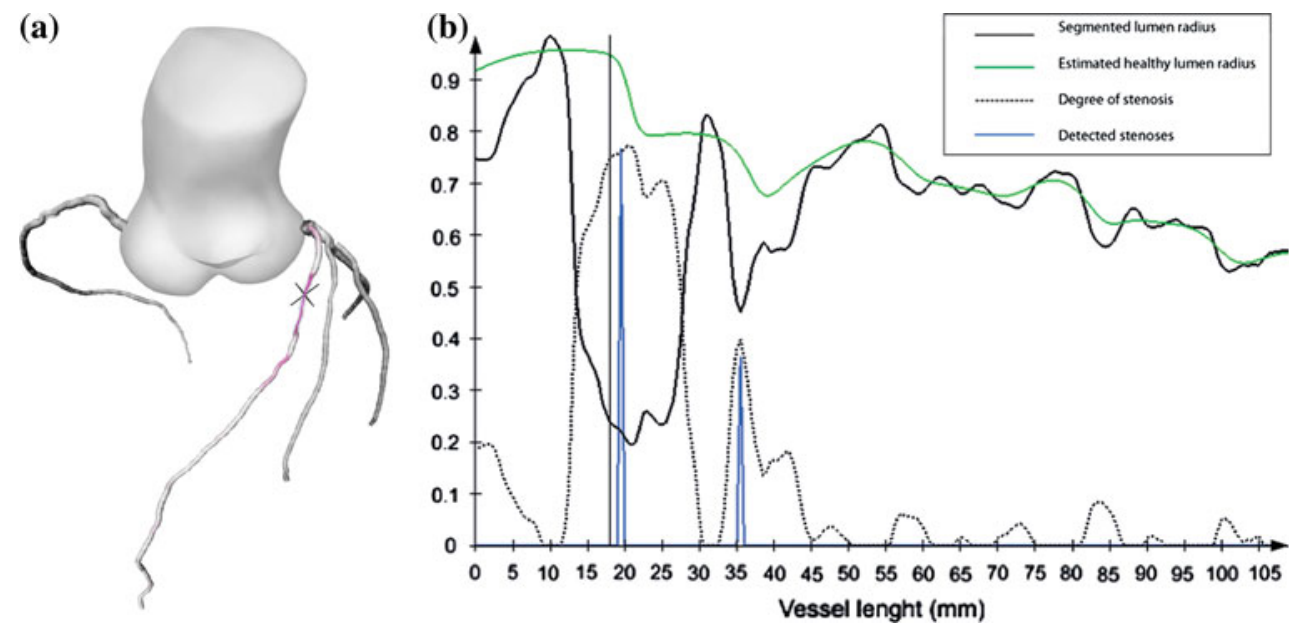

Table 2 Detection-our method's performances compared with the three observers

\begin{tabular}{|c|c|c|c|c|c|c|c|c|}
\hline \multirow[t]{3}{*}{ Method } & \multicolumn{4}{|c|}{ Training (\%) } & \multicolumn{4}{|c|}{ Testing (\%) } \\
\hline & \multicolumn{2}{|l|}{ QCA } & \multicolumn{2}{|l|}{ CTA } & \multicolumn{2}{|l|}{ QCA } & \multicolumn{2}{|l|}{ CTA } \\
\hline & Sens. & PPV & Sens. & PPV & Sens. & PPV & Sens. & PPV \\
\hline Observer 1 & 72 & 49 & 92 & 57 & 86 & 40 & 83 & 61 \\
\hline Observer 2 & 76 & 66 & 82 & 73 & 75 & 51 & 70 & 81 \\
\hline Observer 3 & 52 & 68 & 63 & 74 & 64 & 43 & 66 & 60 \\
\hline Our method & 48 & 63 & 37 & 56 & 29 & 24 & 21 & 23 \\
\hline
\end{tabular}

\section{Results}

Tables 2, 3 and 4 present the training and testing results of our method with respect to the performance of the three observers. The training set consists of 18 datasets and the testing set of 30 datasets.

The ability of a method to discriminate significant stenoses (i.e. stenoses $\geq 50 \%$ ) from non-significant ones was evaluated. Table 2 shows the average results of our method and three observers for stenosis detection measures: sensitivity (Sens.) and positive predictive value (PPV). The results show that on the testing datasets our method obtains a QCA sensitivity of $29 \%$ and a PPV of $24 \%$. With respect to CTA we obtain a sensitivity of $21 \%$ and a PPV of $23 \%$. In general, our results are not as good as the averaged observers' performance (sensitivity of $75 \%$, PPV of $45 \%$ on QCA; sensitivity of $73 \%$, PPV of $67 \%$ on CTA). The ability of our method to discriminate significant stenoses from non-significant ones remains very limited. However, as compared to the current state-of-the art algorithms, our method ranks fourth out of 12 submissions on the test set. 
Table 3 Quantification-our method's performances compared with the three observers

\begin{tabular}{|c|c|c|c|c|c|c|}
\hline \multirow[t]{3}{*}{ Method } & \multicolumn{3}{|l|}{ Training } & \multicolumn{3}{|l|}{ Testing } \\
\hline & \multicolumn{2}{|l|}{ QCA } & \multirow{2}{*}{$\begin{array}{l}\text { CTA } \\
\text { Weighted } \kappa\end{array}$} & \multicolumn{2}{|l|}{ QCA } & \multirow{2}{*}{$\begin{array}{l}\text { CTA } \\
\text { Weighted } \kappa\end{array}$} \\
\hline & Abs diff $(\%)$ & RMS diff $(\%)$ & & Abs diff $(\%)$ & RMS diff (\%) & \\
\hline Observer 1 & 29.7 & 35.1 & 0.71 & 30.1 & 35.2 & 0.74 \\
\hline Observer 2 & 25.5 & 31.8 & 0.84 & 31.1 & 36.5 & 0.77 \\
\hline Observer 3 & 29.1 & 35.1 & 0.73 & 30.6 & 36.9 & 0.73 \\
\hline Our method & 26.3 & 34.8 & 0.37 & 31.0 & 39.3 & 0.29 \\
\hline
\end{tabular}

Table 4 Segmentation-our method's performances as compared to the observers

\begin{tabular}{|c|c|c|c|c|c|c|c|c|c|c|c|c|}
\hline \multirow[t]{3}{*}{ Method } & \multicolumn{6}{|c|}{ Training } & \multicolumn{6}{|c|}{ Testing } \\
\hline & \multicolumn{2}{|c|}{ Dice $(\%)$} & \multicolumn{2}{|c|}{ MSD (mm) } & \multicolumn{2}{|c|}{ MAXSD (mm) } & \multicolumn{2}{|c|}{ Dice (\%) } & \multicolumn{2}{|c|}{ MSD (mm) } & \multicolumn{2}{|c|}{ MAXSD (mm) } \\
\hline & $D$ & $H$ & $D$ & $H$ & $D$ & $H$ & $D$ & $H$ & $D$ & $H$ & $D$ & $H$ \\
\hline Observer 1 & 74 & 79 & 0.26 & 0.26 & 3.29 & 3.61 & 76 & 77 & 0.24 & 0.24 & 2.87 & 3.47 \\
\hline Observer 2 & 66 & 73 & 0.31 & 0.25 & 2.70 & 3.00 & 64 & 72 & 0.34 & 0.27 & 2.82 & 3.26 \\
\hline Observer 3 & 76 & 80 & 0.24 & 0.19 & 3.07 & 3.25 & 79 & 81 & 0.23 & 0.21 & 3.00 & 3.45 \\
\hline Our method & 66 & 70 & 0.37 & 0.32 & 2.49 & 3.04 & 65 & 68 & 0.39 & 0.41 & 2.73 & 3.20 \\
\hline
\end{tabular}

Diseased (D)/healthy (H) segments

Table 3 shows the average results of our method and three observers for stenosis quantification measures. Despite the poor performance of our method to discriminate the non-significant stenoses from significant ones (hard threshold at $50 \%$ ), our method was able to quantify the degree of stenosis as compared to the QCA with an accuracy comparable to the experts. The quantification agreement obtained with the proposed approach as compared to the CTA reference was fair. It should be pointed out that, on the training set $(\kappa=0.37) 90 \%$ of the lesions were estimated in the correct or adjacent class. An averaged absolute difference of $31 \%$, an RMS difference of $39 \%$, and a weighted Kappa value $\kappa=0.29$ were obtained on the test set. The observers (on average) achieved an averaged absolute difference of $31 \%$, an RMS difference of $36 \%$ and $\kappa=0.75$. Our method ranks second out of nine other submissions on the test set.

Table 4 shows the average results of our method and three observers for coronary artery lumen segmentation measures. The similarity between our method and the observers were measured by the Dice similarity index (Dice). The distance between the segmentations was quantified by the root mean squared distance (RMSD) and maximum distance (MAXD). Overall, the Dice and RMSD values obtained on healthy vessel segments were better than the values obtained on diseased segments. The Dice and RMSD were worse than the averaged observers' performance, but the MAXD was better. Figure 9 presents a few examples on longitudinal views of various coronary artery segments. In comparison to those obtained by one of the three observers and the one obtained using our previous approach [31] (i.e. without the calcium suppression step), it can be seen that our segmentation results are very close to the ones obtained by the manual method. Our method ranks second out of six other submissions.

\section{Discussion}

Evaluated algorithm

Although the coronary artery lumen can be automatically segmented with a precision similar to the experts, there is still room for improvement for our stenoses detection/ quantification approach. In the current approach, the stenoses are quantified solely based on the diameter profile of the segmented lumen. Therefore, in case of diffuse disease or long stenoses, the degree of luminal narrowing is generally underestimated. Another possible explanation for the poor performance of our method with respect to stenosis detection could be due to the fact that we assumed the vessels to be cylindrical, hence looked at the problem as reduction in diameter. We could have used an area based stenosis measure as an alternate approach for detection, or measure the minimal diameter in the segmentation for non-circular cross-sections. As the method does not detect a lot of false positives (41 FP's over 48 datasets), it could be used in clinical practice for triage or as a second reader to assist the radiologist. 

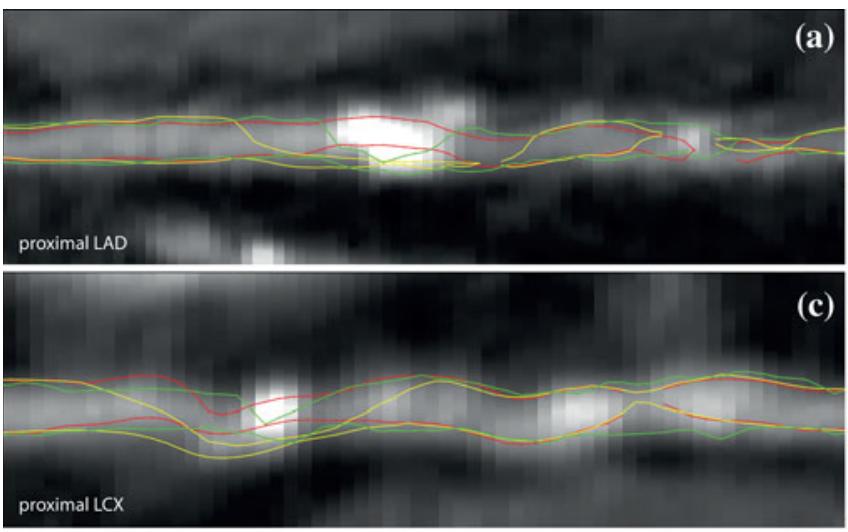

(e)

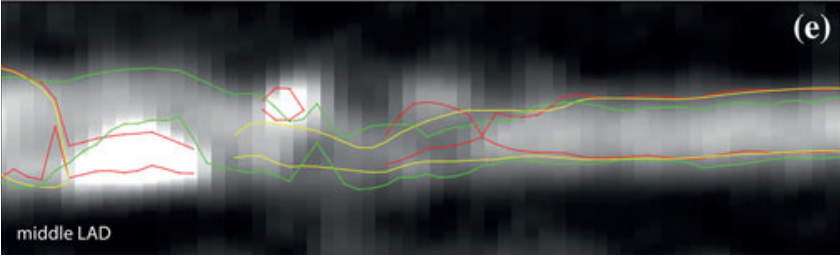

Fig. 9 Coronary artery lumen segmentation examples in CMPR that are based on the manually annotated centerlines. Our previous method (method without the calcium suppression step in the centerline refinement) (red), proposed method (yellow), one of the observers (green). a-c Cases where our method (with the calcium suppression step in the centerline refinement) achieves segmentation similar to the

The relatively low value of the Kappa statistic in the CTA stenoses quantification measure may either be caused by a high number of false positives/negatives or by a high number of lesions reported with more than one grade difference as compared to the CTA reference, or by the linear weights which heavily penalize misclassifications. On the training set, a weighted Kappa value of 0.37 was obtained, and only $10 \%$ of the stenoses had a quantification error of more than one grade (Fig. 8). This highlights that the linearly weighted Kappa is very sensitive to misclassification.

The majority of the stenoses detected with our approach were quantified with an error of only one grade, and most of the misclassifications occured between the mild $(20-50 \%)$ and moderate $(50-70 \%)$ grades. As $50 \%$ is the hard threshold used to discriminate between significant and non-significant lesions, accurate detection of significant stenoses remains a challenge. Considering that our method may be used for triage of patients or as a second reader, the use of a third group "maybe significant", in addition to the significant and non-significant group could be considered, to which all the borderline (40-60\% for instance) detected stenoses are assigned. The radiologists would then have to inspect in more details those stenoses to make a final decision.

The results show that the additional centerline refinement step consisting of calcium suppression from the cost image improves the segmentations compared to our
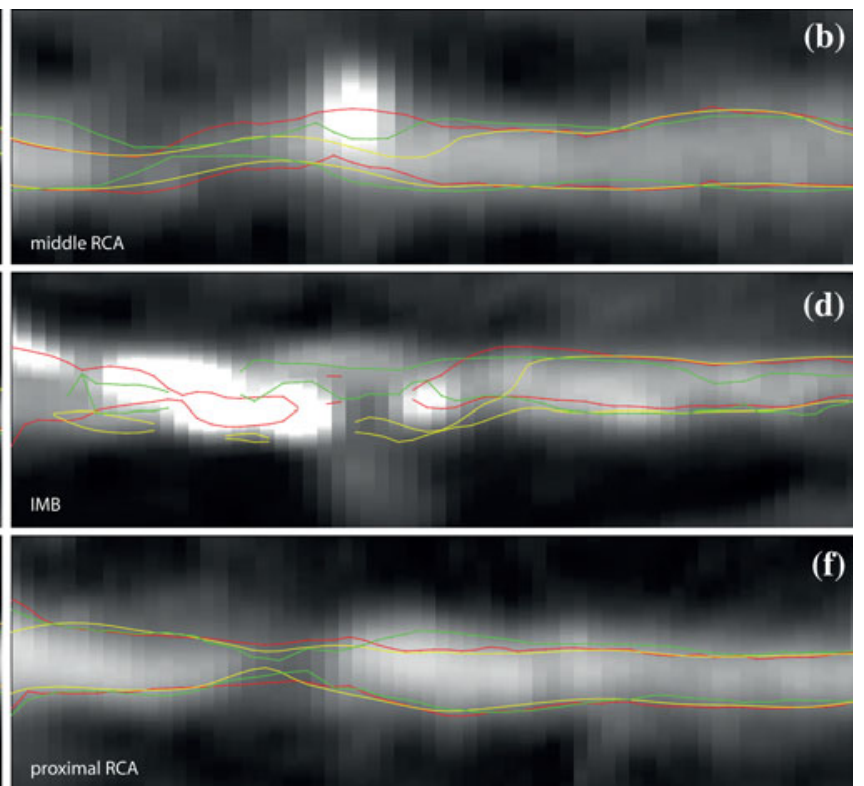

observer. $\mathbf{d}$ Case where the method avoids the calcified plaque and the observer segmented the other side of the plaque. e Case where an issue with large calcified plaque remains. $\mathbf{f}$ Example of segmentation of a coronary segment presenting a soft plaque. Discontinuities in the segmentation, such as the segmentations in $\mathbf{d}$ and $\mathbf{e}$, are a visualization artefact: the segmentation runs out of the CMPR surface

previous approach [31]. Previously, the centerline was attracted to the calcified plaque and therefore, the plaque rather than the vessel was segmented. A simple thresholding technique for removing calcium would not work very efficiently on CTA scans as the intensity of the contrast material between different patients and different vessels is quite dissimilar [22]. Error in estimating a global threshold value for a patient would result in either completely missing medium/small calcium lesions or over segmenting the calcium lesion by including the surrounding contrast material. We chose fitting the intensity profile with a cubic polynomial based on the pilot experiments done on the training data set. Cubic polynomial fitting provided us with a good estimate for a threshold to differentiate between the background contrast intensity and the calcium objects. Higher order polynomials gave us a very smooth fit, making it difficult to estimate the threshold value to separate contrast material from calcium peaks.

The segmentations of the current approach are in better agreement with the observer's ones. The issue with calcified plaques is not completely solved. We were able to prevent the centerlines from running into calcified objects, but for highly calcified regions (Fig. 9d, e), the method may have issues finding the correct lumen. In such cases, our refined centerline tends to run at the very outer border of the lumen, and the derived segmentation is of minimal radius size. However, in such extreme cases, it is not 
always clear how to manually segment the lumen either, and the inter-observer variability is therefore also high.

A limitation of our centerline extraction step is the need to initialize the start and the end points of the coronary arteries. The Coronary Artery Stenoses Detection and Quantification Evaluation Framework organizers provided the participants with the start and the end points of all the vessels that were of interest. For new datasets, the user has to annotate the start and the end point. The initialization process can be simplified by automatically defining the start point in the aorta using the method proposed by [20]. Automatic processing can be further improved by finding the end points using information from an atlas-based coronary density estimate [32]. However, our method can also be used in combination with centerlines that have been automatically obtained [33-36]. The automatically obtained centerline could be used as the initial centerline in our method and subsequently followed by calcium suppression, centerline refinement, lumen segmentation, and stenoses detection and quantification.

\section{Comparison with other evaluated algorithms}

Nine of the eleven other evaluated algorithms were developed following a work-flow similar to ours, consisting of (1) the computation of lumen segmentation, either directly from the input CTA image or using previously extracted centerlines, and (2) the subsequent detection (and quantification) of coronary artery stenoses. Only one of the evaluated algorithms does not involve lumen segmentation, but is using features extracted from the CTA image to detect plaques [37].

To detect and quantify lesions, six out of the nine algorithms estimated a healthy lumen radius by applying various regression approaches to the segmented lumen radius profile (linear for the approaches of [38-42], secondorder for the approach of [43], robust for the approach of [31]). Only in the algorithm proposed by [44], the outer vessel wall was segmented from the CTA image. The remaining two proposed algorithms analyze intensity and geometry features $[45,46]$.

Given an accurate lumen segmentations, our approach outperforms the algorithms proposed by [39] and [44] in the quantification stage, and achieves the best (though fair) quantification agreement as compared to the CTA reference standard. The results thus suggest that robust regression is a good approach to quantify lesions following lumen segmentation. However, there is still room for improvement. Refinement of the stenosis grades using additional morphological and intensity features may lead to improvements in both the detection and quantification steps.

\section{Conclusion}

We presented a method to automatically detect and quantify coronary arteries stenoses, based on coronary artery lumen segmentation. The current results show that the coronary artery lumen can be automatically segmented with a precision similar to the experts. Quantification of the stenoses with respect to the QCA measure can also be performed close to those obtained by the observes. However, automatic discrimination between significant and non-significant lesions in CTA remains a challenge.

Acknowledgments This work is supported by a grant from the Dutch Ministry of Economic Affairs (AgentschapNL) under the title "Het Hart in Drie Dimensies" (PID06003). Medical Delta: Pieken in de Delta.

Conflict of interest None.

\section{References}

1. WHO (2011) Cardiovascular diseases, fact sheet 317. World Health Organization

2. Roger V, Go A, Lloyd-Jones D, Benjamin E, Berry J. Borden, W., Bravata D, Dai S, Ford E, Fox C et al (2012) Heart disease and stroke statistics2012 update a report from the american heart association. Circulation 125(1):e2-e220

3. Fayad Z, Fuster V (2001) Clinical imaging of the high-risk or vulnerable atherosclerotic plaque. Circ Res 89(4):305-316

4. Weustink A, de Feyter P (2011) The role of multi-slice computed tomography in stable angina management: a current perspective. Neth Heart J 19(7-8):336-343

5. Miller JM, Rochitte CE, Dewey M, Arbab-Zadeh A, Niinuma H, Gottlieb I, Paul N, Clouse ME, Shapiro EP, Hoe J et al (2008) Diagnostic performance of coronary angiography by 64-row CT. New Engl J Med 359(22):2324-2336

6. Pugliese F, Hunink M, Gruszczynska K, Alberghina F, Malagó R, van Pelt N, Mollet N, Cademartiri F, Weustink A, Meijboom W et al (2009) Learning curve for coronary CT angiography: what constitutes sufficient training? Radiology 251(2):359-368

7. Melchionna S, Amati G, Bernaschi M, Bisson M, Succi S, Mitsouras D, Rybicki FJ (2013) Risk assessment of atherosclerotic plaques based on global biomechanics. Med Eng Phys 35(9): $1290-1297$

8. Min JK, Leipsic J, Pencina MJ, Berman DS, Koo BK, van Mieghem C, Erglis A, Lin FY, Dunning AM, Apruzzese P et al (2012) Diagnostic accuracy of fractional flow reserve from anatomic CT angiography. Jama 308(12):1237-1245

9. Kirişli H, Schaap M, Metz C, Dharampal A, Meijboom W, Papadopoulou S, Dedic A, Nieman K, de Graaf M, Meijs M, Cramer M, Broersen A, Cetin S, Eslami A, Florez-Valencia L, Lor K, Matuszewski B, Melki I, Mohr B, Oksuz I, Shahzad R, Wang C, Kitslaar P, Unal G, Katouzian A, Orkisz M, Chen C, Precioso F, Najman L, Masood S, Unay D, van Vliet L, Moreno R, Goldenberg R, Vucini E, Krestin G, Niessen W, van Walsum T (2013) Standardized evaluation framework for evaluating coronary artery stenosis detection, stenosis quantification and lumen segmentation algorithms in computed tomography angiography. Med Image Anal 17(8):859-876 
10. Arnoldi E, Gebregziabher M, Schoepf U, Goldenberg R, RamosDuran L, Zwerner P, Nikolaou K, Reiser M, Costello P, Thilo C (2010) Automated computer-aided stenosis detection at coronary CT angiography: initial experience. Eur Radiol 20(5):1160-1167

11. Halpern E, Halpern D (2011) Diagnosis of coronary stenosis with CT angiography: comparison of automated computer diagnosis with expert readings. Acad Radiol 18(3):324-333

12. Khan M, Wesarg S, Gurung J, Dogan S, Maataoui A, Brehmer B, Herzog C, Ackermann H, Aßmus B, Vogl, T (2006) Facilitating coronary artery evaluation in MDCT using a 3D automatic vessel segmentation tool. Eur Radiol 16(8):1789-1795

13. Kelm B, Mittal S, Zheng Y, Tsymbal A, Bernhardt D, VegaHiguera F, Zhou S, Meer P, Comaniciu D (2011) Detection, grading and classification of coronary stenoses in computed tomography angiography. Medical Image Computing and Computer-Assisted Intervention-MICCAI, pp 25-32

14. Wesarg S, Khan M, Firle E (2006) Localizing calcifications in cardiac CT data sets using a new vessel segmentation approach. J Digit Imaging 19(3):249-257

15. Xu Y, Liang G, Hu G, Yang Y, Geng J, Saha P (2012) Quantification of coronary arterial stenoses in CTA using fuzzy distance transform. Comput Med Imaging Graph 36(1):11-24

16. Saur S, Alkadhi H, Desbiolles L, Székely G, Cattin P (2008) Automatic detection of calcified coronary plaques in computed tomography data sets. Medical Image Computing and ComputerAssisted Intervention-MICCAI, pp 170-177

17. Tessmann M, Vega-Higuera F, Fritz D, Scheuering M, Greiner G (2009) Multi-scale feature extraction for learning-based classification of coronary artery stenosis. In: SPIE medical imaging, International Society for Optics and Photonics, pp 726002-726002

18. Zuluaga MA, Magnin IE, Hoyos MH, Leyton EJD, Lozano F, Orkisz M (2011) Automatic detection of abnormal vascular crosssections based on density level detection and support vector machines. Int J Comput Assist Radiol Surg 6(2):163-174

19. Agatston A, Janowitz W, Hildner F, Zusmer N, Viamonte Jr M, Detrano R (1990) Quantification of coronary artery calcium using ultrafast computed tomography. J Am Coll Cardiol 15(4):827

20. Metz C, Schaap M, Weustink A, Mollet N, van Walsum T, Niessen W (2009) Coronary centerline extraction from ct coronary angiography images using a minimum cost path approach. Med Phys 36(12):5568-5579

21. Frangi A, Niessen W, Vincken K, Viergever M (1998) Multiscale vessel enhancement filtering. Medical Image Computing and Computer-Assisted Intervention-MICCAI, pp 130-137

22. Rybicki FJ, Otero HJ, Steigner ML, Vorobiof G, Nallamshetty L, Mitsouras D, Ersoy H, Mather RT, Judy PF, Cai T et al (2008) Initial evaluation of coronary images from 320-detector row computed tomography. Int J Cardiovasc Imaging 24(5):535-546

23. Steigner ML, Mitsouras D, Whitmore AG, Otero HJ, Wang C, Buckley O, Levit NA, Hussain AZ, Cai T, Mather RT et al. (2010) Iodinated contrast opacification gradients in normal coronary arteries imaged with prospectively ECG-gated single heart beat 320-detector row computed tomography. Circ Cardiovasc Imaging 3(2):179-186

24. Tang H, Walsum T, van Onkelen RS, Hameeteman R, Klein S, Schaap M, Tori FL, van den Bouwhuijsen QJ, Witteman J, der Lugt A et al. (2012) Semiautomatic carotid lumen segmentation for quantification of lumen geometry in multispectral MRI. Med Image Anal 16:1201-1215

25. Gülsün MA, Tek H (2008) Robust vessel tree modeling. In: Medical Image Computing and Computer-Assisted Interventation-MICCAI, Springer, pp 602-611

26. Carreira-Perpinan M (2007) Gaussian mean-shift is an EM algorithm. Pattern Anal Mach Intell IEEE Trans 29(5):767-776

27. van Walsum T, Schaap M, Metz C, van der Giessen A, Niessen W (2008) Averaging centerlines: mean shift on paths. Medical
Image Computing and Computer-Assisted Intervention-MICCAI, pp 900-907

28. Dijkstra E (1959) A note on two problems in connexion with graphs. Numerische mathematik 1(1):269-271

29. Schaap M, Neefjes L, Metz C, van der Giessen A, Weustink A, Mollet N, Wentzel J, van Walsum T, Niessen W (2009) Coronary lumen segmentation using graph cuts and robust kernel regression. In: Information processing in medical imaging. Springer, pp 528-539

30. Debruyne M, Hubert M, Suykens J (2008) Model selection in kernel based regression using the influence function. J Mach Learn Res 9:2377-2400

31. Shahzad R, van Walsum T, Kirişli H, Tang H, Metz C, Schaap M, van Vliet L, Niessen W (2012) Automatic detection, quantification and lumen segmentation of the coronary arteries using two-point centerline extraction scheme. In: Proceedings of MICCAI workshop "3D cardiovascular imaging: a MICCAI segmentation challenge"

32. Shahzad R, Schaap M, van Walsum T, Klien S, Weustink AC, van Vliet LJ, Niessen WJ (2010) A patient-specific coronary density estimate. In: Biomedical imaging: from nano to macro, 2010 IEEE international symposium on, IEEE, pp 9-12

33. Kitamura Y, Li Y, Ito W (2012) Automatic coronary extraction by supervised detection and shape matching. In: Biomedical imaging (ISBI), 2012 9th IEEE international symposium on, IEEE, pp 234-237

34. Yang G, Kitslaar P, Frenay M, Broersen A, Boogers MJ, Bax JJ, Reiber JH, Dijkstra J (2012) Automatic centerline extraction of coronary arteries in coronary computed tomographic angiography. Int J Cardiovasc Imaging 28(4):921-933

35. Goldenberg R, Eilot D, Begelman G, Walach E, Ben-Ishai E, Peled N (2012) Computer-aided simple triage (CAST) for coronary CT angiography (CCTA). Int J Comput Assist Radiol Surg 7(6):819-827

36. Zambal S, Hladuvka J, Kanitsar A, Bühler K (2008) Shape and appearance models for automatic coronary artery tracking. Insight $\mathrm{J} 4: 1-8$

37. Duval M, Ouzeau E, Precioso F, Matuszewski B (2012) Coronary artery stenoses detection with random forest. In: Proceedings of MICCAI workshop "3D cardiovascular imaging: a MICCAI segmentation challenge"

38. Cetin S, Unal G (2012) Automatic detection of coronary artery stenosis in CTA based on vessel intensity and geometric features. In: Proceedings of MICCAI workshop "3D cardiovascular imaging: a MICCAI segmentation challenge"

39. Broersen A, Kitslaar P, Frenay M, Dijkstra J (2012) FrenchCoast: fast, robust extraction for the nice challenge on coronary artery segmentation of the tree. In: Proceedings of MICCAI workshop "3D cardiovascular imaging: a MICCAI segmentation challenge"

40. Flórez Valencia L, Orkisz M, Corredor Jerez, RA, Torres González JS, Correa Agudelo EM, Mouton C, Hernández Hoyos M (2012) Coronary artery segmentation and stenosis quantification in CT images with use of a right generalized cylinder model. In: Proceedings of MICCAI workshop "3D cardiovascular imaging: a MICCAI segmentation challenge"

41. Mohr B, Masood S, Plakas C (2012) Accurate stenosis detection and quantification in coronary CTA. In: Proceedings of MICCAI workshop "3D cardiovascular imaging: a MICCAI segmentation challenge"

42. Öksüz d, Ünay D, Kadipaşaoğlu K (2012) A hybrid method for coronary artery stenosis detection and quantification. In: Proceedings of MICCAI workshop "3D cardiovascular imaging: a MICCAI segmentation challenge"

43. Eslami A, Aboee A, Hodaei Z, Moghaddam MJ, Carlier S, Katouzian A, Navab N (2012) Quantification of coronary arterial stenosis by inflating tubes in CTA images. In: Proceedings of 
MICCAI workshop "3D cardiovascular imaging: a MICCAI segmentation challenge"

44. Wang C, Moreno R, Smedby Ö (2012) Vessel segmentation using implicit model-guided level sets. In: Proceedings of MICCAI workshop "3D cardiovascular imaging: a MICCAI segmentation challenge"

45. Lor K, Chen C (2012) Probabilistic model based evaluation of coronary artery stenosis on CTA. In: Proceedings of MICCAI workshop "3D cardiovascular imaging: a MICCAI segmentation challenge"

46. Melki I, Talbot H, Cousty J, Pruvot C, Knoplioch J, Launay L, Najman L (2012) Automatic coronary arteries stenoses detection in 3D CTA. In: Proceedings of MICCAI workshop "3D cardiovascular imaging: a MICCAI segmentation challenge" 